\title{
Sequential multiphoton multiple ionization of atomic argon and xenon irradiated by X-ray free-electron laser pulses from SACLA
}

\author{
K Motomura ${ }^{1}$, H Fukuzawa ${ }^{1,2}$, S-K Son ${ }^{3}$, S Mondal $^{1}$,

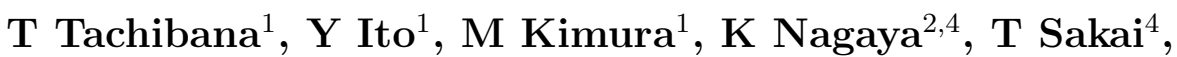 \\ K Matsunami ${ }^{4}$, S Wada $^{2,5}$, H Hayashita ${ }^{5}$, J Kajikawa ${ }^{5}$, \\ X-J Liu ${ }^{6}, \mathbf{R}$ Feifel $^{7}$, P Johnsson $^{8}$, M Siano $^{9}$, E Kukk ${ }^{10}$, \\ B Rudek ${ }^{11,12}$, B Erk ${ }^{11,12}$, L Foucar ${ }^{11,13}$, E Robert ${ }^{6}$, C Miron ${ }^{6}$, \\ K Tono ${ }^{14}$, Y Inubushi ${ }^{2}$, T Hatsui ${ }^{2}, \mathbf{M}$ Yabashi $^{2}, \mathbf{M}$ Yao $^{4}$, \\ R Santra ${ }^{3,15}$ and K Ueda ${ }^{1,2}$ \\ ${ }^{1}$ Institute of Multidisciplinary Research for Advanced Materials, Tohoku University, \\ Sendai 980-8577, Japan \\ ${ }^{2}$ RIKEN SPring-8 Center, Kouto 1-1-1, Sayo, Hyogo 679-5148, Japan \\ ${ }^{3}$ Center for Free-Electron Laser Science (CFEL), DESY, 22607 Hamburg, Germany \\ ${ }^{4}$ Department of Physics, Kyoto University, Kyoto 606-8502, Japan \\ ${ }^{5}$ Department of Physical Science, Hiroshima University, Higashi-Hiroshima 739-8526, \\ Japan \\ ${ }^{6}$ Synchrotron SOLEIL, L'Orme des Merisiers, Saint-Aubin, BP 48, FR-91192 \\ Gif-sur-Yvette Cedex, France \\ ${ }^{7}$ Department of Physics and Astronomy, Uppsala University, P.O. Box 516, SE-751 \\ 20 Uppsala, Sweden \\ ${ }^{8}$ Department of Physics, Lund University, P.O. Box 118, 22100 Lund, Sweden \\ ${ }^{9}$ Blackett Laboratory, Imperial College London, London SW7 2AZ, United Kingdom \\ ${ }^{10}$ Department of Physics and Astronomy, University of Turku, 20014, Finland \\ ${ }^{11}$ Max-Planck Advanced Study Group at CFEL, 22607 Hamburg, Germany \\ ${ }^{12}$ Max-Planck-Insitut für Kernphysik, 69117 Heidelberg, Germany \\ ${ }^{13}$ Max-Planck-Insitut für medizinische Forschung, 69120 Heidelberg, Germany \\ ${ }^{14}$ Japan Synchrotron Radiation Research Institute (JASRI), Kouto 1-1-1, Sayo, \\ Hyogo 679-5198, Japan \\ ${ }^{15}$ Department of Physics, University of Hamburg, 20355 Hamburg, Germany \\ E-mail: fukuzawa@tagen.tohoku.ac.jp \\ E-mail: sangkil.son@cfel.de
}

\begin{abstract}
We have investigated multiphoton multiple ionization of argon and xenon atoms at $5 \mathrm{keV}$ using a new X-ray free electron laser (XFEL) facility, the SPring-8 Angstrom Compact free electron LAser (SACLA) in Japan. The experimental results are compared with new theoretical results presented here. The absolute fluence of the XFEL pulse has been determined with the help of the calculations utilizing twophoton processes in the argon atom. The high charge states up to +22 observed for Xe in comparison with the calculations point to the occurrence of sequential $L$-shell multiphoton absorption and of resonance-enabled X-ray multiple ionization.
\end{abstract}




\section{Introduction}

X-ray free-electron lasers (XFELs) provide extremely intense femtosecond X-ray pulses that promise access to high-resolution structure determination, while outrunning any radiation damage. Serial femtosecond X-ray crystallography (SFX), which integrates thousands of diffraction patterns of XFEL pulses scattered on a serial flow of microcrystals, is in the process of revolutionizing molecular structure determination $[1$, $2,3]$. Last year, one of the milestones was achieved, namely the determination of the unknown structure of a protein by SFX, which is expected to help fighting sleeping sickness [4]. The phase problem, however, still remains a major obstacle to structural reconstruction from SFX data in general. Phasing SFX data has so far relied on molecular replacement $[1,2,3,4]$. If the structure is completely unknown, phasing approaches usually make use of anomalous dispersion of scattering signals from specific heavy atoms as reference scatterers. One of the established methods along this line is a multi-wavelength anomalous diffraction method (MAD). Electronic damage due to extremely strong X-ray pulses [5], however, may question the application of the conventional MAD to SFX data. Thus, a FEL-specific high-intensity MAD has been proposed by some of the present authors [6]. The proposed method, as well as related methods that make use of anomalous dispersion in intense X-ray fields, requires the ability to describe the dynamical behavior of heavy atoms in high-intensity X-ray pulses. This description is challenging and thus both, further theoretical development and benchmark experimental data, are urgently needed to take a step forward towards addressing the phase problem in SFX.

In early 2012, a new XFEL facility, the SPring-8 Angstrom Compact free electron LAser (SACLA) [7], started user operation in Japan. One of the first experiments at SACLA was to detect anomalous signals of the naturally occurring sulfur atoms in a protein (lysozyme microcrystal) at a photon energy of $7.3 \mathrm{keV}$, set far from the sulfur $\mathrm{K}$ edge $(\sim 2.5 \mathrm{keV})$ to suppress the electronic damage [8]. Though attempts at solving the anomalous substructure failed, the results demonstrated that anomalous data can indeed be obtained by SFX. As a next step, we have investigated dynamical behaviors, i.e., deep inner-shell multiphoton ionization and cascade decay, of heavy atoms Ar and Xe exposed to high-intensity X-ray pulses at 5 and $5.5 \mathrm{keV}$. In the present paper, we describe the details of our experimental and theoretical methods using the $5 \mathrm{keV}$ data. The results at $5.5 \mathrm{keV}$ are reported elsewhere [9].

Multiphoton processes are well-known phenomena in the optical regime and have been investigated for decades. The advent of extreme ultraviolet (EUV) FELs [10, 11] with femtosecond pulse widths has led to renewed interests in multiphoton ionization processes in the EUV regime. We refer here only to some of the latest works on atoms carried out at FLASH [10] in Germany [12, 13, 14] and at the SCSS test accelerator [11] in Japan $[15,16,17,18,19,20]$. The motivation for these studies was mainly to reveal the pathways of the multiphoton multiple ionization newly opened up by these light sources. X-ray FEL pulses that became available at LCLS in the USA [21] had a huge impact 
(a)

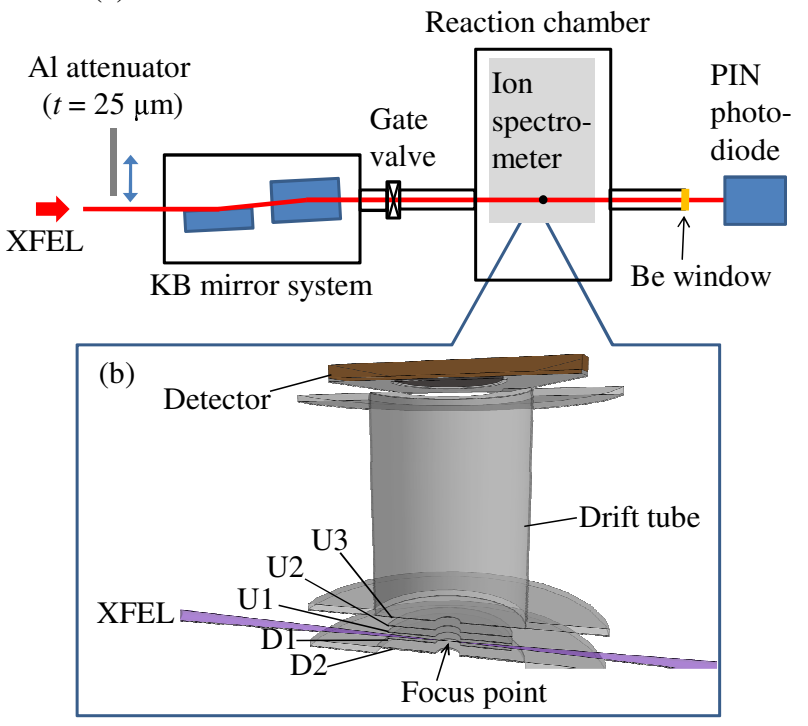

Figure 1. (Color online) (a) Experimental configuration. (b) Cross section of the ion spectrometer.

on this field [22, 23, 24, 25]. So far, however, the investigations have been limited to the photon energy range up to $2 \mathrm{keV}$, i.e., the upper photon energy limit of the AMO beam line [26] at the LCLS [21]. Multiphoton processes at higher photon energies discussed in the present work are of particular importance because of their direct relevance for the electronic damage in high-resolution x-ray imaging measurements, as described above.

The present paper is organized in the following manner. The next section, section 2 , describes our experimental setup, as well as the experimental procedures and the data analysis. Section 3 describes our theoretical developments. Section 4 presents results and discussions, while Section 5 concludes the present work.

\section{Experiment}

\subsection{Configuration}

The experiment has been carried out at the experimental hutch 3 (EH3) of beam line 3 (BL3) at SACLA in Japan [7]. The photon energy was set to $5 \mathrm{keV}$. The photon band width was $\sim 60 \mathrm{eV}$ (FWHM). The repetition rate of the XFEL pulses was $10 \mathrm{~Hz}$. The pulse width has not been measured experimentally, but was estimated to be in the range between 10 and 30 fs (FWHM) [27].

Figure 1 (a) shows the experimental configuration. A Kirkpatrick-Baez (KB) mirror system is permanently installed at EH3 [28]. The focal length (work distance) is $\sim 1.4 \mathrm{~m}$. The XFEL beam is focused by the KB mirror system to a focal size of $\sim 1 \mu \mathrm{m}$ (FWHM) in diameter. The Rayleigh length is $\sim 8 \mathrm{~mm}$. The sample gas (Ar or Xe) was introduced to our ultrahigh-vacuum reaction chamber by a pulsed supersonic gas jet [29] crossing the focal point of the XFEL pulses. The inner diameter of the gas jet nozzle was $0.25 \mathrm{~mm}$. 
The jet was skimmed by the two skimmers placed at 20 and $400 \mathrm{~mm}$ from the nozzle. The inner diameters of the 1 st and 2nd skimmers were 0.5 and $2 \mathrm{~mm}$, respectively. The distance between the 2 nd skimmer and the reaction point was $250 \mathrm{~mm}$. The gas beam at the reaction point was estimated to be $\sim 2 \mathrm{~mm}$ (FWHM) in diameter. Thus the source volume of the ions was roughly of cylindrical shape of $\sim 1 \mu \mathrm{m}$ in diameter and $\sim 2 \mathrm{~mm}$ along the XFEL beam. After crossing the gas jet at right angles, the XFEL beam exits the vacuum chamber via a beryllium window. The relative X-ray pulse energy was measured shot-by-shot by a p-intrinsic-n (PIN) photodiode, after the pulse energy was reduced by aluminum sheets of $0.2 \mathrm{~mm}$ thickness so that the linear response of the photodiode was assured. The shot-by-shot pulse energy fluctuation was $\pm 25 \%(50 \%$ FWHM).

\subsection{Ion spectrometry}

Figure 1 (b) depicts the ion spectrometer. The XFEL beam crosses the supersonic gas jet in the region between the U1 and D1 electrodes. Ions produced by the XFEL irradiation are extracted towards the upper direction by an electrostatic gradient field produced by five electrodes labelled D2, D1, U1, U2 and U3. The distances between D2-D1, D1-U1, $\mathrm{U} 1-\mathrm{U} 2$ and $\mathrm{U} 2-\mathrm{U} 3$ are 15, 10, 10 and $15 \mathrm{~mm}$, respectively. The diameter of the hole of the D2 electrode is $22 \mathrm{~mm}$, and those of the D1, U1, U2 and U3 electrodes are $20 \mathrm{~mm}$. The thickness of these electrodes is $1 \mathrm{~mm}$ each. The hole of the U3 electrode is covered with a mesh to terminate the gradient field.

After passing the mesh, ions fly through a $339 \mathrm{~mm}$ field free region and arrive at a detector that consists of microchannel plates (MCP) and a delay-line anode (Roentdek HEX80) [30]. Signals from the MCP and the delay-line anode were fed into an 8-channel digitizer. The wave forms recorded by the digitizer were analyzed by a software discriminator [31] and the arrival time and the arrival position of each ion were determined. This procedure minimized the dead-time of the ion detection. The voltages on the spectrometer were tuned for the best mass resolution, i.e., the voltages of 520, 341, 281 and $281 \mathrm{~V}$ were applied to the D2, D1, U1 and U2 electrodes, respectively, while the U3 electrode and drift tube were grounded. With these field conditions the image of the source volume was expected to be expanded by a factor of five according to the simulation.

The simulation of ion trajectories shows that the TOF slightly depends on the ion arrival position on the detector, as a consequence of different departure positions perpendicular to the spectrometer's axis. A compensation on the measured TOF is introduced to improve the mass resolution further as shown in figure 2, where the TOF of the $\mathrm{Xe}^{9+}$ isotopes versus the arrival positions in the $\mathrm{X}$ direction on the detector are plotted before (a) and after (b) compensation of the TOF. The X direction is the direction of the XFEL beam propagation. The lengths of the image confirm that the image is indeed expanded by a factor of about five. Figure 2 (c) and (d) depict projections of (a) and (b), respectively. We can clearly see an improvement of the mass 


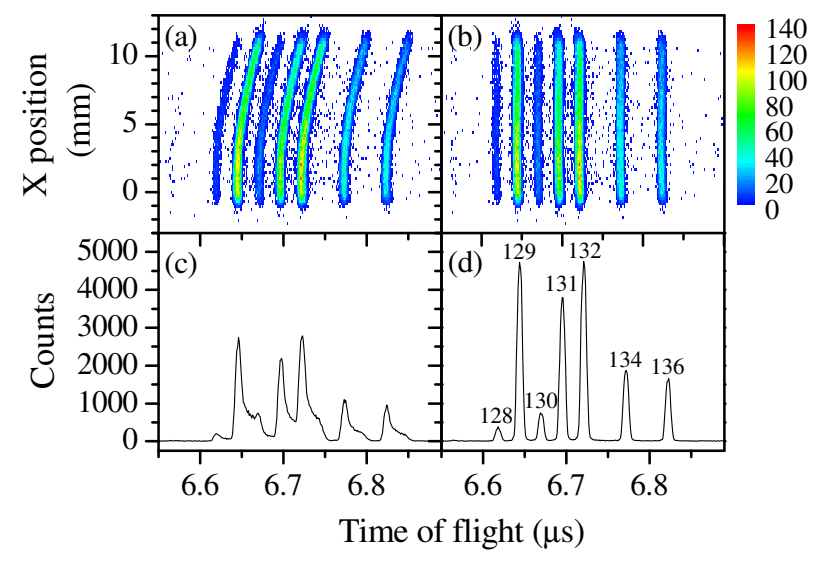

Figure 2. Plots of ion time of flight versus arrival $\mathrm{X}$ position on the detector before (a) and after (b) compensation of the time of flight for seven isotopes of $\mathrm{Xe}^{9+}$. (c) and (d) show the projections of (a) and (b), respectively. The numbers in (d) indicate the mass of the Xe isotopes in atomic mass units.

resolution after compensation of the TOF. Seven isotopes, ${ }^{128} \mathrm{Xe},{ }^{129} \mathrm{Xe},{ }^{130} \mathrm{Xe},{ }^{131} \mathrm{Xe}$, ${ }^{132} \mathrm{Xe},{ }^{134} \mathrm{Xe}$ and ${ }^{136} \mathrm{Xe}$, are well-resolved.

Although we obtained enough resolution to resolve isotopes for all the observed charge states of Xe, certain ions having very close mass-to-charge ratios but different charge states cannot be separated completely. For example, as an ultimate case, ${ }^{128} \mathrm{Xe}^{16+}$ and ${ }^{136} \mathrm{Xe}^{17+}$ cannot be separated in principle because these ions have the same massto-charge ratio. In order to extract the ratios of the charge states in such cases, we have employed least squares fit to the overlapped part of the charge states, using literature values of the natural abundance of xenon isotopes. Since most peaks were well-separated and only a small portion of the peaks were overlapped, uncertainties stemming from the fitting were negligibly small.

\subsection{Counting statistics}

In ion spectrometry described in the previous subsection, several ions may hit the detector within a small area almost simultaneously. In such cases, signals from the MCP detector, as well as the readout signals from the delay-line anode, are merged in a single pulse. Even though our ion detection procedure minimizes the dead-time [31], we still encountered the situation where we could not use the usual counting mode in the Ar multiple ionization measurements, because Ar has a single isotope and thus there is only a single line in the TOF spectrum for each charge state. In comparison, Xe has many lines as shown in figure 2. Also the charge distribution of $\mathrm{Ar}$ is much narrower, up to charge state of +10 , in comparison with the charge distribution of Xe up to +22 . To obtain ion charge distributions in such situations, we used the following algorithm based on the Poisson distribution. Namely, the probability to detect $n$ ions per FEL 

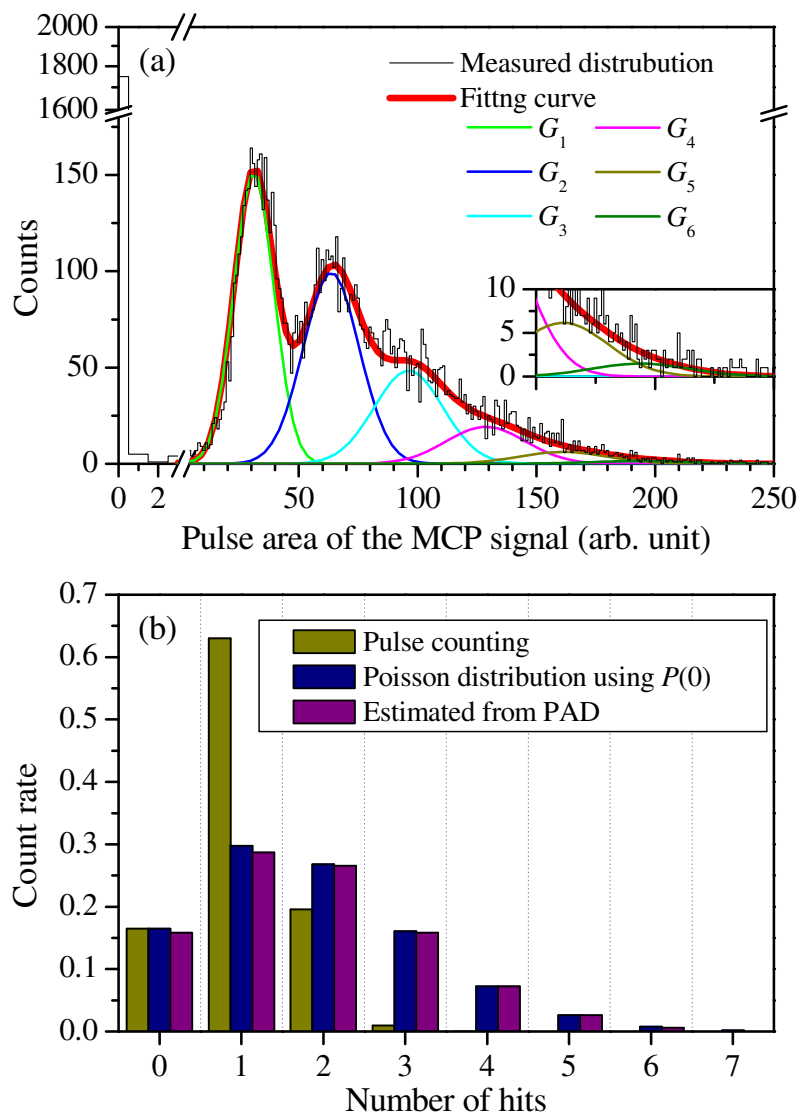

Figure 3. (a) The pulse area distribution of the MCP signals for $\mathrm{Ar}^{4+}$ detection and the fitting result. On the left, the graph is zoomed in to 0 showing the number of events for "detecting no ion". (b) Comparison between the pulse counting, the Poisson distribution constructed by "detecting no ion" and the fitting for the "pulse area" distribution of the MCP read-out signals for $\mathrm{Ar}^{4+}$ detection.

pulse, $P(n)$, follows the Poisson distribution:

$$
P(n)=\frac{e^{-\lambda} \lambda^{n}}{n !}
$$

where $\lambda$ is an average value of $n$, i.e., the average number of the events to detect a specific charge state (charge state of +4 in case of figure 3) per FEL pulse. Substitution of $n=0$ gives

$$
\lambda=\ln \left(\frac{1}{P(0)}\right) .
$$

Thus, we can obtain the average number of events detecting a specific charge state per FEL pulse from the average number of events of "detecting no ion" at the specific charge state over many FEL shots. If we counted the number of detected ions at the specific charge state by the conventional counting method in our experimental condition corresponding to figure 3 (a), the number of events for "detecting more than one ion" would be suppressed and the number of events of "detecting one ion" would be enhanced. As a result, the average number of the detected ions per FEL pulse would 
be underestimated. On the other hand, counting events of the "detecting no ion" would not suffer from pile-up. However, the background signal must be negligible in order to count "detecting no ion" events over many FEL shots. Thus, to avoid contaminations from the background signals, the signals to be counted are to be strictly gated by the expected arrival time and the arrival position for each charge state.

To confirm that the method of counting events for "detecting no ion" works as expected, let us examine the distribution of the number of ion detections using the "pulse area" distribution (PAD) shown in figure 3 (a). Here the "pulse area" means the integrated signal, within the expected TOF range for a specific charge state of the ion, read out from the MCP by the digitizer. If $k$ ions hit the MCP almost simultaneously, we may detect one pile-up pulse formed by the unresolved $k$ pulses. The area of the pile-up pulse by the $k$ ions may be on average approximately $k$ times the pulse area of detecting one ion. Let us approximate that the PAD of the MCP signals by detecting $k$ ions is represented by the Gaussian distribution $G_{k}$ and that the mean and variance of $G_{k}$ are $k$ times these of $G_{1}$. Figure $3(\mathrm{a})$ depicts the PAD of the MCP signal for Ar ${ }^{4+}$ detection. The result of fitting, $\sum G_{k}(k=1-6)$ and each Gaussian component, $G_{1}-G_{6}$, are also shown in figure $3(\mathrm{a})$. The fitting curve represents the measured PAD very well and allows us to estimate the distribution for the number of hits (detected ions).

Figure 3(b) shows the distribution for the number of $\mathrm{Ar}^{4+}$ ions per FEL pulse obtained from three different methods, (1) the pulse counting using both the MCP and the delay-line signals [31], (2) the Poisson distribution constructed by "detecting no ion" and (3) the fitting of the PAD for the MCP readout signals in figure 3(a). While methods (2) and (3) agree very well, method (1) underestimates the number of the events. From this comparison, we can conclude that method (2), which was used in Ref. [9] and will be used in the present paper, is free from erroneous counting due to pile-up and thus that the ion charge distributions obtained by method (2) should be reasonably accurate.

\section{Theory}

The electronic response to an XFEL pulse is well characterized by a sequence of onephoton ionizations accompanied by decay processes $[22,33]$. To describe this sequential multiphoton multiple ionization dynamics, we employ the XАTOM toolkit [32]. Using the Hartree-Fock-Slater method, we calculate atomic data, i.e., photoionization cross sections, Auger and Coster-Kronig rates and fluorescence rates, for all possible multiplehole electronic configurations that may be formed by removing zero, one, or more electrons from the ground-state configuration of the neutral atom. The calculated atomic data serve as input parameters for a set of coupled rate equations for the timedependent populations of the configurations. The number of all possible configurations is corresponding to the matrix size for the set of rate equations and the total number of atomic data is related to the complexity of constructing the rate-equation matrix. For Ar, we use the approach described in Ref. [32], adding shakeoff processes within 
the sudden approximation [34]. The number of configurations for Ar is 1,323 and the number of atomic data at $5 \mathrm{keV}$ is 41,192 .

For heavy atoms, however, the ionization dynamics become complex, not only because the heavy atom has many electrons to be ionized, but also because it has many ionization channels involving complex inner-shell decay cascade. Therefore, the straightforward sequential ionization model for heavy atoms becomes challenging because of too many configurations and too many atomic data. For example, Xe $M$-shell ionization dynamics requires 1,120,581 coupled rate equations and 42,433,458 atomic data at $2 \mathrm{keV}$ [24]. We have addressed the former issue of too large matrix size to be solved by introducing a Monte Carlo approach in Refs. [24, 35], where all atomic data were pre-calculated. For Xe $L$-shell ionization dynamics considered here, however, the complexity is further increased, so $\sim 2 \times 10^{7}$ coupled rate equations with $\sim 2 \times 10^{9}$ atomic data would need to be solved. In this case, all calculations of those atomic data are too expensive. Therefore, we have extended XАTOM by applying the Monte Carlo procedure for both calculating atomic data and searching probable ionization pathways [9]. When a certain configuration is visited during an ionization pathway chosen by the Monte Carlo sampling, atomic data are calculated for the corresponding configuration. In this way, atomic data are computed only when they are required. This extension enables us to treat ionization dynamics of heavy atoms with no limit of configurational space. Saving in computational time is dramatic. For example, one calculation of Xe at $5 \mathrm{keV}$ and $200 \mu \mathrm{J} / \mu \mathrm{m}^{2}$ takes only 1 day using one CPU on the laboratory workstation, which would take more than 3 years if all atomic data had to be calculated.

In the present calculations, the photon energy is fixed at $5 \mathrm{keV}$. The pulse shape is assumed to be a Gaussian of 30 fs (FWHM). In the regime of sequential multiphoton absorption, the results are not sensitive to the pulse shape or spikiness of individual pulses [33]. We also did the calculation for $10 \mathrm{fs}$ (FWHM) at a single XFEL fluence and confirmed that the results are in good agreement. About $1 \times 10^{5}$ to $2 \times 10^{5}$ trajectories are performed to achieve convergency of $10^{-5}$ for all charge-state populations. All calculated results presented here are obtained by 3 -dimensional integration [24] over the interaction volume of $1 \mu \mathrm{m} \times 1 \mu \mathrm{m} \times 2 \mathrm{~mm}$ (all lengths are given in FWHM), according to the instrumental configuration described in subsection 2.1.

\section{Results and discussion}

\subsection{Two-photon multiple ionization of Ar}

Figure 4 compares the experimental charge state distribution of Ar at $5 \mathrm{keV}$ as derived by the method in subsection 2.3 and the theoretical results described in section 3 . The theoretical charge state distribution is scaled so that the sum of the individual charge state yields is equal to the total ion yield in the experiment. At the photon energy of $5 \mathrm{keV}$, the $K$-shell electrons of Ar can be ionized and direct two-photon ionization and resonant excitation are not relevant. We find that the theoretical distributions up to the 


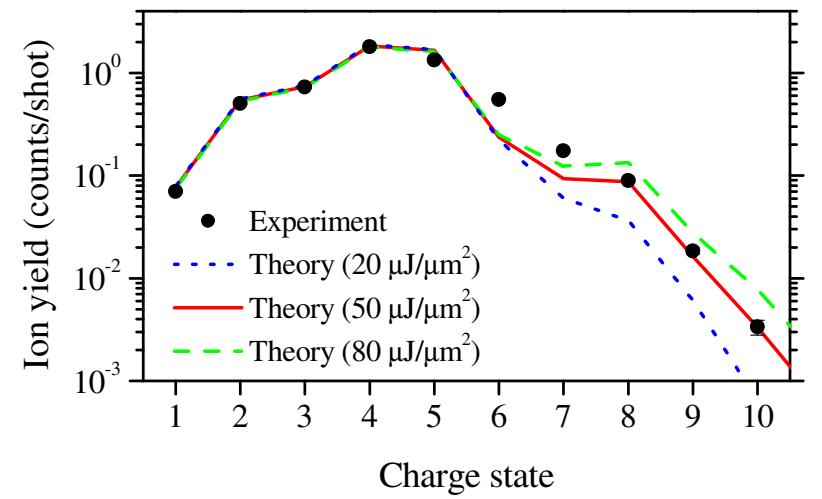

Figure 4. (Color online) Experimental and theoretical charge state distributions of Ar at the photon energy of $5 \mathrm{keV}$.

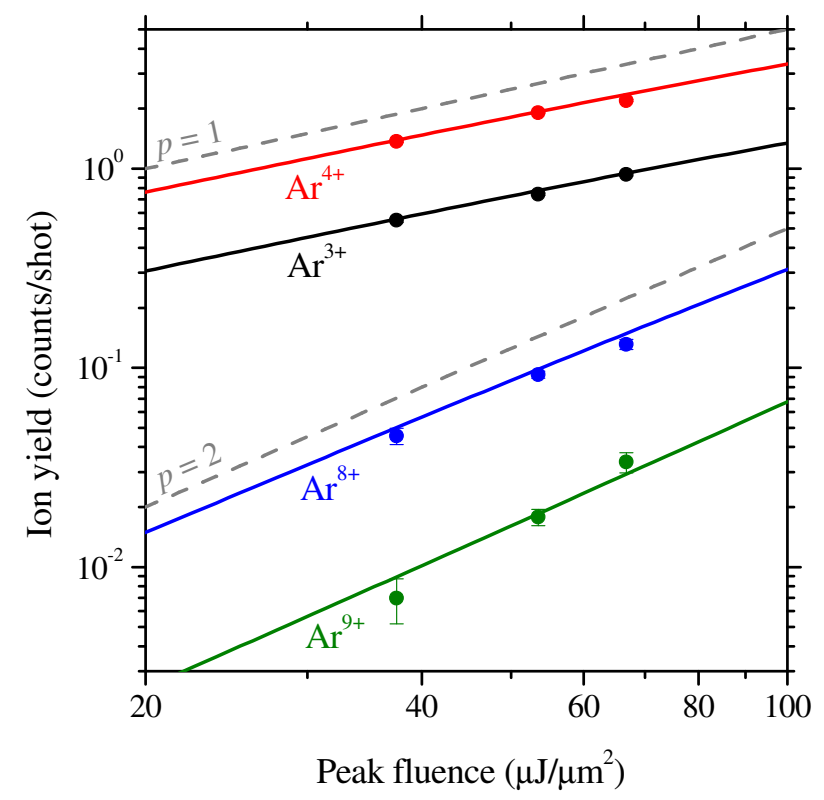

Figure 5. (Color online) XFEL fluence dependence of the ion yields for $\operatorname{Ar}^{n+}(n=3$, 4, 8 and 9). Closed circles with error bars depict the experimental results and solid lines depict the theoretical results. Lines with slope $p=1$ and 2 are also shown as broken lines to guide the eye.

charge state of +6 do not vary when varying the XFEL fluence, illustrating that these ions are produced by single photon absorption. In general, they are in good agreement with the experiment, except that in theory the $\mathrm{Ar}^{5+}$ yield is slightly overestimated while the yields of $\mathrm{Ar}^{6+}$ and $\mathrm{Ar}^{7+}$ are underestimated. This discrepancy may be attributed to the non-relativistic treatment and lack of double Auger processes [36]. Further, we find that both the experimental and theoretical yields of $\mathrm{Ar}^{8+}$ and $\mathrm{Ar}^{9+}$ depend quadratically on the XFEL fluence, which is shown in figure 5.

In order to compare the theoretical and experimental results for Xe in the next subsection, we need to obtain the peak fluence of the XFEL pulse employed for our 
experiment. The fluence at the center of the X-ray beam focus defines the peak fluence $F_{\text {peak }}$, which is given by

$$
F_{\text {peak }}=\frac{4 \ln 2}{\pi} \times \frac{E}{A} \times T
$$

where $E$ is the nominal pulse energy from the monitor located upstream of the beam line, $T$ is the transmission between the monitor and the focal point, $A$ is the focal area given in FWHM $\times$ FWHM and the coefficient of $4 \ln 2 / \pi$ comes from the assumed Gaussian focal shape. We estimated relative contributions from two- and one-photon processes using the ratio of $R \equiv\left(Y_{8+}+Y_{9+}\right) /\left(Y_{3+}+Y_{4+}\right)$, where $Y_{n+}$ is the yield of $\mathrm{Ar}^{n+}$. Fitting the theoretical value of $R$ to the experimental one by the least squares method, we have obtained $53 \mu \mathrm{J} / \mu \mathrm{m}^{2}$ for the peak fluence during the Ar measurements. Note that the experimental results in Fig. 4 are ensemble-averaged results measured at the full XFEL pulse energy that shows the shot-by-shot fluctuation. In order to make comparison with the theoretical results and determine the absolute peak fluence, we asuumed $\left\langle Y_{n+}[F]\right\rangle \approx Y_{n+}[\langle F\rangle]$, where $F$ is the fluence and $\langle\cdot\rangle$ denotes an ensemble average over shots. This assumption of the averaged radiation pulse including the pulse energy fluctuation is well valid in the regime of sequential ionization dynamics [33]. Therefore, the pulse energy fluctuation should not considerably affect the peak fluence determination.

Figure 5 depicts the yields of the individual charge states as a function of the peak fluence of the XFEL pulse. To obtain several data points with respect to the peak fluence, we binned the data using the relative XFEL fluence measured by the PIN photodiode (see figure 1). All theoretical ion yields in figure 5 are scaled by a single factor such that the yield of $\mathrm{Ar}^{4+}$ is matched with that in the experiment at $\sim 53 \mu \mathrm{J} / \mu \mathrm{m}^{2}$. The yields of $\mathrm{Ar}^{8+}$ and $\mathrm{Ar}^{9+}$ show the quadratic dependence on the XFEL fluence, illustrating two-photon absorption during the XFEL pulse.

\subsection{Multiphoton multiple ionization of Xe}

Figure 6(a) depicts the charge state distribution of Xe at the full XFEL pulse energy. The peak fluence determined via the calibration using Ar and the in situ relative fluence measurement by the PIN photodiode was $47 \mu \mathrm{J} / \mu \mathrm{m}^{2}$. We also recorded the charge state distribution at a pulse energy attenuated by an aluminum foil of $25 \mu \mathrm{m}$ thickness located upstream of the KB mirror system (see figure 1). The attenuated pulse energy was $27 \%$ of the full pulse energy according to the reading of the PIN photodiode. The charge state distribution at the $13 \mu \mathrm{J} / \mu \mathrm{m}^{2}$ is shown in the figure $6(\mathrm{~b})$. The charge state distribution varies as the peak fluence varies. We also compare the theoretical charge state distributions with the experiment at the same peak fluences used in the experiment. The experimental and theoretical results are in reasonable agreement up to the charge state of +15 . In theory, there is a projecting peak at $\mathrm{Xe}^{7+}$ (and accompanying discrepancies for +8 to +11 ), which may come from missing Auger (Coster-Kronig) channels at $\mathrm{Xe}^{7+}$ due to the non-relativistic treatment. More noticeable discrepancy 


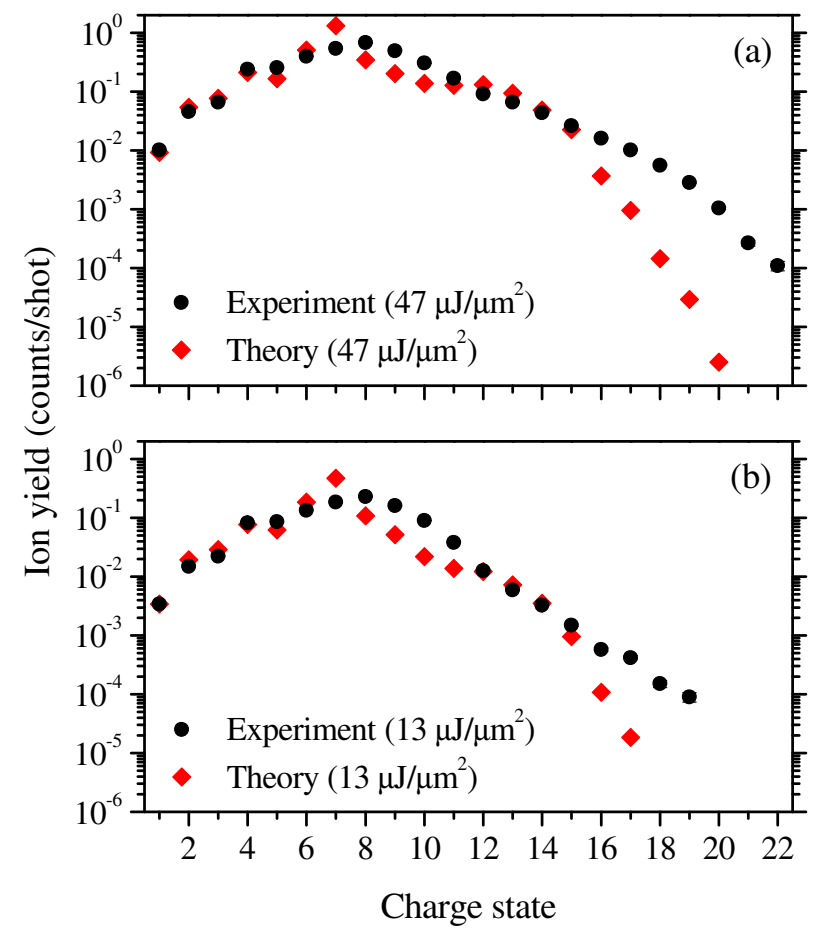

Figure 6. (Color online) Experimental and theoretical charge state distributions of $\mathrm{Xe}$ at the photon energy of $5 \mathrm{keV}$ for the full XFEL pulse energy (a) and $27 \%$ of the full pulse energy (b).

between experiment and theory is above the charge state of +16 , which increases with increasing the charge state. The discrepancy is larger than that shown in the case of $5.5 \mathrm{keV}$ photon energy [9].

At a photon energy of $5 \mathrm{keV}$, the $2 p$ electrons of neutral Xe are ionized. According to our calculations, the $2 p$ ionization thresholds become larger than $5 \mathrm{keV}$ beyond $\mathrm{Xe}^{11+}$, so the sequential one-photon ionization ends at $\mathrm{Xe}^{11+}$. As a result, the theoretical charge state distribution shows a small hump at $\mathrm{Xe}^{12+}$ and a strong decrease for higher charge states. Note that the current calculations do not include relativistic effects. The experimental binding energies of $2 s, 2 p_{1 / 2}$ and $2 p_{3 / 2}$ of neutral Xe are $5.5 \mathrm{keV}$, $5.1 \mathrm{keV}$ and $4.8 \mathrm{keV}$, respectively, whereas the Hartree-Fock-Slater calculations give $5.1 \mathrm{keV}$ and $4.8 \mathrm{keV}$ for $2 s$ and $2 p$ binding energies, respectively. Thus, the calculated $2 p$ ionization cross section of neutral Xe at $5 \mathrm{keV}$ is overestimated by a factor of $3 / 2$. Therefore, if the relativistic effects were taken into account, the theoretical yields would be even lowered and the discrepancy between experiment and theory would be more pronounced. Bearing in mind this fact, we interpret that this discrepancy, significantly higher ion yields for high charge states and a highest charge production of $\mathrm{Xe}^{22+}$ in the experiment, is a signature of the resonance-enabled X-ray multiple ionization (REXMI) mechanism $[24,25]$, where transient resonant excitation enhances the ionization process beyond the limit expected by the straightforward sequential ionization model. This feature was not shown in the previous experiment at $5.5 \mathrm{keV}$ [9], because $5.5 \mathrm{keV}$ is still 


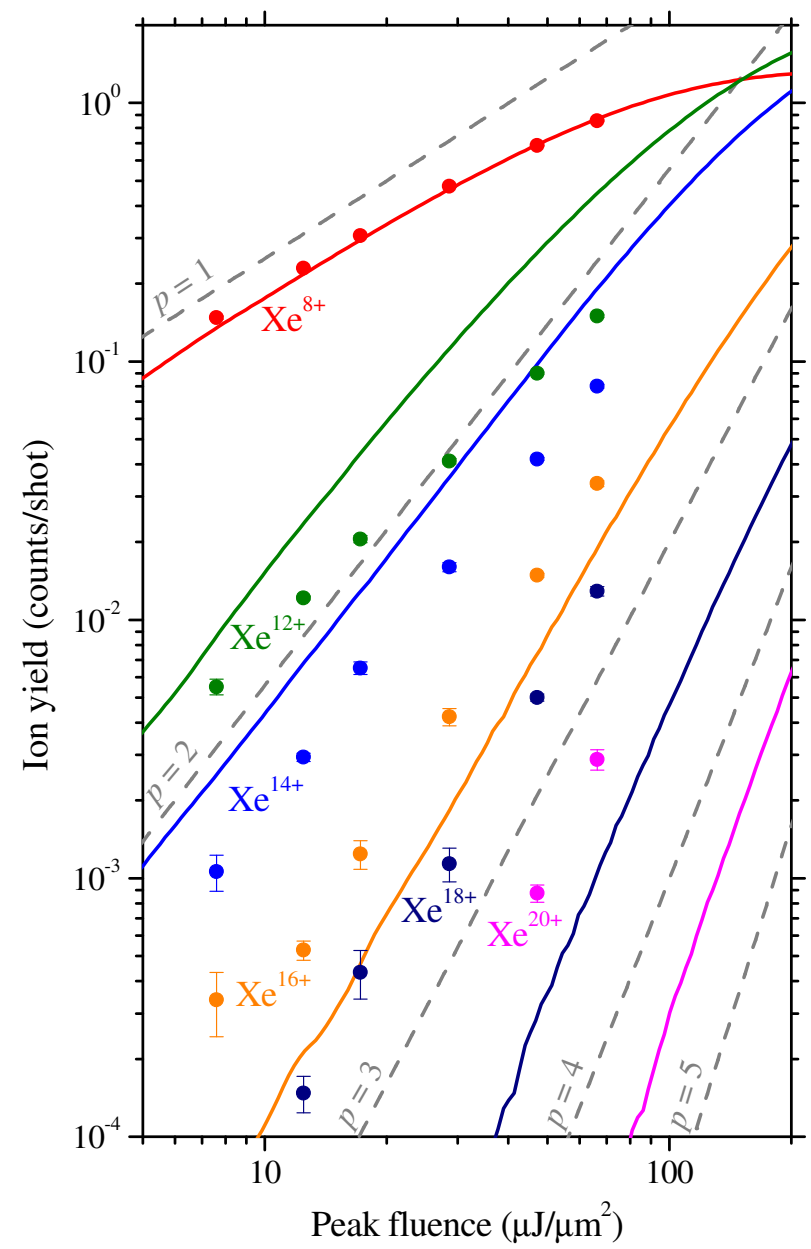

Figure 7. (Color online) XFEL fluence dependence of the ion yields for $\mathrm{Xe}^{n+}(n=8$, 12, 14, 16, 18 and 20). Closed circles with the error bars depict the experimental results and solid lines depict the theoretical results. Lines with slope $p=1,2,3,4$ and 5 are also shown as broken lines to guide the eye. The uncertainty of the peak fluence is expected to be $\sim \pm 10 \%$.

above the $L$-shell ionization thresholds of the charge states up to $\mathrm{Xe}^{23+}$ and the XFEL fluence was not high enough to populate those high charge states $(>+23)$ to initiate REXMI. The present results suggest an exsitence of REXMI at a higher photon energy than was available at the LCLS experiments.

In figure 7 , the ion yields for $\mathrm{Xe}^{n+}(n=8,12,14,16,18$ and 20$)$ are plotted as a function of the peak fluence. To obtain several data points with respect to the peak fluence, we first merged the results measured at two different pulse energies and then re-binned the data. Straight lines with a slope of $1,2,3,4$ and 5 are also shown as a guide to the eye. All theoretical ion yields are scaled by a single factor such that the yield of $\mathrm{Xe}^{8+}$ is matched with that in the experiment at $\sim 47 \mu \mathrm{J} / \mu \mathrm{m}^{2}$. The yield of $\mathrm{Xe}^{8+}$ exhibits a slope of less than one, illustrating that this ion is produced by single-photon absorption and that the single-photon absorption process is close to saturation. For higher charge states, the ion yields exhibit a non-linear dependence on the XFEL fluence 
and their slopes increase with increasing the charge state, indicating the occurrence of multiphoton absorption.

\section{Conclusions}

We have investigated multiphoton multiple ionization of $\mathrm{Ar}$ and $\mathrm{Xe}$ atoms utilizing the XFEL facility SACLA. We have demonstrated that position sensitivity for the ion detection in an ion TOF spectrometer improves the mass resolution significantly and that counting statistics based on the Poisson distribution helps to increase the count rates to shorten the acquisition time that is often necessary for FEL experiments. We have confirmed the occurrence of the sequential $K$-shell two-photon absorption of $\mathrm{Ar}$ and used it to determine the absolute fluence of the XFEL pulses with the help of benchmark ab initio calculations. We have confirmed the occurrence of the sequential $L$-shell multiphoton absorption of Xe and a signature of REXMI for the first time in the hard X-ray regime.

The present X-ray photon energy and the heavy atoms are of particular relevance to the molecular structure determination with XFELs. Understanding of ionization dynamics of heavy atoms exposed to high intensity high-energy X-ray beams provides useful input for future molecular imaging experiments using XFELs.

\section{Acknowledgments}

The experiments were performed at SACLA with the approval of JASRI and the program review committee (No. 2012A8036). The authors are grateful to A. Rudenko, D. Rolles and J. Ullrich for discussion. This study was supported by the X-ray Free Electron Laser Utilization Research Project and the X-ray Free Electron Laser Priority Strategy Program of the Ministry of Education, Culture, Sports, Science and Technology of Japan (MEXT), by the Japan Society for the Promotion of Science (JSPS) KAKENHI Grant Numbers 21244062, 23241033, by MEXT KAKENHI Grant Number 22740264, by the Proposal Program of SACLA Experimental Instruments of RIKEN, by the Cooperative Research Program of Network Joint Research Center for Materials and Devices of MEXT, and by the IMRAM project. S.M. acknowledges financial support from JSPS. R.F. acknowledges financial support from the Swedish Research Council (VR). P.J. acknowledges support from the Swedish Research Council (VR) and the Swedish Foundation for Strategic Research (SSF).

\section{References}

[1] H. N. Chapman et al., Nature (London) 470, 73 (2011).

[2] S. Boutet et al., Science 337, 362 (2012).

[3] L. C. Johansson, Nature Methods 9, 265 (2012).

[4] L. Redecke, K. Nass et al., Science 339, 227 (2013).

[5] H. M. Quiney and K. A. Nugent, Nature Phys. 7, 142 (2011). 
[6] S.-K. Son, H. N. Chapman and R. Santra, Phys. Rev. Lett. 107, 218102 (2011).

[7] T. Ishikawa et al., Nature Photon. 6, 540 (2012).

[8] T. R. M. Barends et al., Acta Cryst. D 69, 838 (2013).

[9] H. Fukuzawa et al., Phys. Rev. Lett. 110, 173005 (2013).

[10] W. Ackermann et al., Nature Photon. 1, 336 (2007).

[11] T. Shintake et al., Nature Photon. 2, 555 (2008).

[12] M. Richter et al., Phys. Rev. Lett. 102, 163002 (2009).

[13] M. Kurka et al., New J. Phys. 12, 073035 (2010).

[14] A. Rouzee et al., Phys. Rev. A 83, 031401(R) (2011).

[15] K. Motomura et al., J. Phys. B: At. Mol. Opt. Phys. 42, 221003 (2009).

[16] H. Fukuzawa et al., J. Phys. B: At. Mol. Opt. Phys. 43, 111001 (2010).

[17] Y. Hikosaka et al., Phys. Rev. Lett. 105, 133001 (2010).

[18] T. Sato et al., J. Phys. B: At. Mol. Opt. Phys. 44, 161001 (2011).

[19] E. V. Gryzlova et al., Phys. Rev. A 84, 063405 (2011).

[20] A. Hishikawa et al., Phys. Rev. Lett. 107, 243003 (2011).

[21] P. Emma et al., Nature Photon. 4, 641 (2010).

[22] L. Young et al., Nature (London) 466, 56 (2010).

[23] G. Doumy et al., Phys. Rev. Lett. 106, 083002 (2011).

[24] B. Rudek et al., Nature Photon. 6, 858 (2012).

[25] B. Rudek et al., Phys. Rev. A 87, 023413 (2013).

[26] J. D. Bozek, Eur. Phys. J. Spec. Top. 169, 129 (2009).

[27] Y. Inubushi et al., Phys. Rev. Lett. 109, 144801 (2012).

[28] H. Yumoto et al., Nature Photon. 7, 43 (2013).

[29] K. Nagaya et al., J. Electron Spectrosc. Relat. Phenom. 181, 125 (2010).

[30] O. Jagutzki et al., IEEE Trans. Nucl. Sci. 49, 2477 (2002).

[31] K. Motomura et al., Nucl. Instrum. Methods Phys. Res., Sect. A 606, 770 (2009).

[32] S.-K. Son, L. Young and R. Santra, Phys. Rev. A 83, 033402 (2011).

[33] N. Rohringer and R. Santra, Phys. Rev. A 76, 033416 (2007).

[34] A. G. Kochur, A. I. Dudenko, V. L. Sukhorukov and I. D. Petrov, J. Phys. B: At. Mol. Opt. Phys. 27, 1709 (1994).

[35] S.-K. Son and R. Santra, Phys. Rev. A 85, 063415 (2012).

[36] A. M. El-Shemi and Y. A. Lotfy, Eur. Phys. J. D 32, 277 (2005). 